\title{
Socioeconomic status of pregnancy outcomes in Chongqing, Southwest China
}

\section{Ting Zhang}

Chongqing Medical University

\section{Wei Zhou}

Chongqing Health Center for Women and Children

\section{Qiang Tan}

Chongqing Medical University

\section{Ping Tang}

Chongqing Medical University

\section{Tao Kuang}

Chongqing Medical University

\section{Rui-Yuan Zhang}

Chongqing Medical University

\section{Yu-Ting Chen}

Chongqing Medical University

Yin-Yin Xia ( $\nabla 100118 @ c q m u . e d u . c n)$

Chongqing Medical University

\section{Ting-Li Han}

Chongqing Medical University

\section{Chen Chang}

Chongqing Medical University

\section{Hong-Bo Qi}

the First Affiliated Hospital of Chongqing Medical University

\section{Hua Zhang}

the First Affiliated Hospital of Chongqing Medical University

\section{Philip N Baker}

University of Leicester

\section{Research article}

Keywords: Pregnancy outcomes, Socioeconomic status, Maternal education level, Participant and partner's income, Maternal occupation

Posted Date: October 31st, 2019 
DOI: https://doi.org/10.21203/rs.2.16639/v1

License: (c) (1) This work is licensed under a Creative Commons Attribution 4.0 International License. Read Full License 


\section{Abstract}

Background: The relationship between socioeconomic status (SES) and pregnancy outcomes has rarely been studied in Southwest China. Our aim was to investigate whether SES was associated with the risk of adverse pregnancy outcomes.

Methods: Between 2015 and 2017, we analyzed data from 1273 women in Chongqing, China, enrolled in the Complex Lipids in Mothers and Babies (CLIMB) trial in which mothers received complex lipid supplementation during pregnancy. Information on SES (maternal educational level, participant and partner's income and maternal occupation) was collected during face-to-face interviews using standard questionnaires. Pregnancy outcomes considered were gestational diabetes mellitus (GDM), premature rupture of membrane (PROM), cesarean section (C-section), preterm birth (PTB), macrosomia, low birth weight (LBW), large for gestational age (LGA), and small for gestational age (SGA). Logistic regression models were used to estimate odds ratios (ORs) and 95\% confidence intervals (Cls) for pregnancy outcomes in relation to SES. Supplementation had no effect on pregnancy outcomes, so our analysis did not subdivide our participants on this basis.

Results: Women who were managers or professionals were less likely to suffer from macrosomia and LGA. After adjustment for potential confounders, the ORs for macrosomia and LGA were $0.44(95 \% \mathrm{Cl}$ : $0.21,0.95)$ and $0.48(95 \% \mathrm{Cl}: 0.26,0.89)$, respectively. Other pregnancy outcomes were not affected by maternal occupation. Moreover, no significant relationships were observed between the other two SES indicators (participant and partner's income and maternal education) and adverse pregnancy outcomes.

Conclusions: Our findings suggested that maternal occupation was a more reliable predictor of pregnancy outcomes than maternal education and participant and partner's income.

\section{Background}

Pregnancy outcomes, including gestational diabetes mellitus (GDM), premature rupture of membrane (PROM), cesarean section (C-section), preterm birth (PTB), macrosomia, low birth weight (LBW), large for gestational age (LGA), and small for gestational age (SGA), have serious health consequences across the life course for both mothers and their offspring. Adverse pregnancy outcomes are determined by a complex interplay of genetic and environmental factors. One potential environmental parameter is socioeconomic status (SES), also termed as socioeconomic position (SEP) or social class, which refers to the social and economic factors that reflect the positions and prestige individuals or groups hold within the structure of a society [1].

There is a growing literature that explores the influence of SES factors on pregnancy outcomes. Some studies have shown that low SES in pregnant women can increase the risk of adverse pregnancy outcomes such as GDM, PTB, LBW, and SGA [2-7]. However, others have suggested that SES indicators have little influence on pregnancy outcomes [7-11]. These contradictory findings may reflect differences between countries, regions, or races. 
China has witnessed a remarkable and rapid social-economic growth in the last few decades; investments in education and healthcare have increased accordingly [12,13], but economic, education and healthcare development varies greatly by different regions of China. To our best knowledge, there are few, if any, investigations of the association between SES and pregnancy outcomes in Southwest China. Therefore, we examined the associations of three measures of SES maternal educational level, participant and partner's income, and maternal occupation with pregnancy outcomes in urban areas of Chongqing, the largest of the four direct-controlled municipalities in China.

\section{Methods}

\section{Study participants}

The study participants $(n=1500)$ were enrolled during September 2015 to June 2017 into the CLIMB study at the First Affiliated Hospital of Chongqing Medical University or Chongqing Health Centre for Women and Children in China; details of the CLIMB study have been described elsewhere[14]. Women who withdrew from the study $(n=146)$, whose pregnancies were terminated $(n=29)$, who miscarried $(n=$ $12)$, or were lost to follow up $(n=40)$, were excluded from the analysis. 1273 women were thus included in the final analysis.

\section{Data collection and diagnostic criteria}

All participants were interviewed by experienced trained nurses at enrollment. Information on sociodemographic factors (maternal age, ethnicity, marital status, maternal education level, participant and partner's income and maternal occupation), maternal anthropometry (BMI at enrollment), recreational drug/alcohol use before or during pregnancy, smoking before or during pregnancy and any pertinent gynecological and obstetric (gravidity, delivery, abortion, infertility) history was collected during this process. Maternal gestational week was determined by LMP and <mid-trimester ultrasound information; (if the two estimates differed by over 7 days, gestational age was based on the obstetric ultrasound data)

Three indicators were used to define SES: maternal educational level, participant and partner's income, and maternal occupation. In our study, maternal educational level was based on total years of schooling and divided into three groups: low educational level ( $<12$ years), medium educational level (12-17 years), high educational level ( $>17$ years). Participant and partner's income was combined and stratified into four categories: low income (<7000 yuan/month), middle income (7000-10000 yuan/month), middle-high income (10000-16000 yuan/month), high income (>16000 yuan/month). Maternal occupation was classified according to the International Standard Classification of Occupations standard (ISCO-08), and then divided into three groups: no occupation or student, workers/technicians/clerical/service occupations and managers/professionals.

\section{Diagnostic criteria for pregnancy outcomes}


Pregnancy outcomes were managed by obstetricians and abstracted from the medical records.

Pregnancy outcomes included: gestational diabetes mellitus (GDM), premature rupture of membrane (PROM), cesarean section (C-section), preterm birth (PTB), macrosomia, low birth weight (LBW), large for gestational age (LGA), small for gestational age (SGA). The diagnostic criteria are shown in Table 1.

\section{Statistical analysis}

Data analysis was performed using SPSS (version 19.0, IBM, USA). A value of $P<0.05$ was considered significant. Data were described as mean \pm SD for continuous variables or as percentage for categorical variables. Logistic regression models were used to examine the associations between SES (maternal educational level, participant and partner's income and maternal occupation) and risk of pregnancy outcomes. The results are presented as odds ratios (ORs) and $95 \%$ confidence intervals (Cls), using low educational level (12 years or less), $<7000 y u a n / m o n t h$ (low income) or no occupation or student as the reference groups. In the multivariable logistic model I, we adjusted for maternal age (continuous), early pregnancy BMI (continuous), Han nationality (yes or no), primiparity (yes or no), history of abortion (yes or no), gestational age at delivery (continuous), newborn sex (female or male). GDM, PIH, PE, PROM, Csection and PTB was not adjusted for gestational age at delivery and newborn sex. In the multivariable logistic model II, we additionally adjusted for the other SES parameters. We did not adjust for maternal smoking and alcohol use during pregnancy, because only five women reported smoking or alcohol use during pregnancy among 1273 women included in the analysis. The presence of multicollinearity was adjudged if tolerance values were less than 0.4 and the variance inflation (VIF) was greater than 2.5 using Allison's criteria [15].

\section{Results}

The demographic characteristics, socioeconomic status and prevalence of pregnancy outcomes are shown in Table 2. The mean age of women was $28.7 \pm 3.6$ years, $98.7 \%$ were Han nationality and about $77.5 \%$ were primiparous. The mean early pregnancy BMl was $21.5 \pm 2.9 \mathrm{~kg} / \mathrm{m}^{2}$. The mean gestational age at birth was $39.4 \pm 1.5$ weeks, and the mean birth weight was $3310.7 \pm 436.9 \mathrm{~g}$. The majority of women had university degree (79.7\%), participant and partner's income in the middle level (36.8\%), and had occupation as workers, technicians, clerks and service (48.6\%). Approximately $37.2 \%$ of pregnant women had C-Section, $25.5 \%$ of women affected by GDM and PROM occurred $24.6 \%$ in the study. Supplementation had no effect on pregnancy outcomes, so our analysis did not subdivide our participants on this basis (This paper is under review at Scientific Report).

To examine the association between maternal educational level and risk of pregnancy outcomes, logistic regression models were used (Table 3). Compared with women with low educational level (12 years or less), those with medium educational level (12-17 years) were associated with higher risk of PROM, the ORs of PROM were 1.88 (95\% Cl: 1.13, 3.13) and $1.73(95 \% \mathrm{Cl}: 1.03,2.91)$ for those with medium educational level (12-17 years), in the unadjusted model and model I, respectively. However, in the model 
II, no significant association were observed between education and PROM. Furthermore, no significant association between maternal educational level and other pregnancy outcomes were observed using any of the univariate and multivariable models.

Table 4 presents the ORs and $95 \%$ Cls for pregnancy outcomes in association with participant and partner's income. Only C-section was found to be associated with middle-high income of pregnant women (10000-16000yuan/month), as compared with low income of pregnant women (<7000yuan/month) (OR: $0.72 ; 95 \% \mathrm{Cl}: 0.52,0.99)$. The differences remained statistically significant (OR: $0.70 ; 95 \% \mathrm{Cl}: 0.50,0.97)$ after adjusting for the mothers' age, BMI, Han nationality, primiparity, history of abortion, but non-significance once there was further adjustment for the mothers' education and occupation (OR: $0.75 ; 95 \% \mathrm{Cl}: 0.53,1.06)$. There were no significant correlations between maternal and partner's income and any of the other adverse pregnancy outcomes.

Table 5 shows the association between maternal occupation and pregnancy outcomes. A significantly correlation between occupation and macrosomia and LGA was observed. Women who were managers or professionals had a lower risk of macrosomia and LGA. The adjusted ORs of macrosomia was 0.44 ( $95 \%$ $\mathrm{Cl}: 0.21,0.95)$ for women were managers or professionals compared to those who had no occupation or who were students. In addition, the adjusted ORs of LGA was $0.48(95 \% \mathrm{Cl}: 0.26,0.89)$ for women who were managers or professionals compared to those who had no occupation or who were students. No significant associations between maternal occupation and the other pregnancy complications were observed.

The results of coefficiency statistics for multicollinearity are shown in Additional file 1 . The estimation of the tolerance value and VIF in the model was greater than 0.4 and less than 2.5 , respectively, which revealed that there was no multicollinearity in the multivariate logistic model.

\section{Discussion}

This study investigated the association between SES and risks of adverse pregnancy outcomes in Chongqing. Our study highlights the influence of maternal occupation on macrosomia and LGA; women who were managers or professionals had lower risks of macrosomia and LGA after adjustment for potential confounders. No significant relationship were observed between maternal occupation and other pregnancy outcomes. The other two indicators of SES (maternal educational level and participant and partner's income) did not have effect on pregnancy outcomes after adjustment for confounders. As in previous studies that the associations between SES and pregnancy outcomes depended on which SES parameter was used $[9,16,17]$. A systematic review on socioeconomic disparities in adverse birth outcomes demonstrated that different measures may capture different aspects of relative or absolute socioeconomic advantage, which may vary in their importance for birth outcomes [18]. For instance, education levels reflect obtained knowledge and skills-related assets of an individual, income captures a person's material resources and social standing, while occupation is reflection of a person's place in society related to situations linked to working conditions [1]. 
Macrosomia and LGA have become frequent clinical challenges in obstetrics and neonatology, and are associated with many maternal and neonatal complications $[19,20]$. In our study, the incidence of macrosomia and LGA were $5.3 \%$ and $9.1 \%$, respectively. These rates are low compared to two recent studies reported in China, which found the incidence of macrosomia to be $8.7 \%$ of births [21] and of LGA to be $9.9 \%$ [22]. The associations between parameters of SES and PTB, LBW and SGA are established [5, 23-25]; in contrast, few studies have explored the influence of SES on macrosomia and LGA, and the results have been inconsistent. A large population-based study from Shaanxi province found no association between SES and the risk of macrosomia, although the rates of macrosomia were higher in pregnancies associated with high SES [9]. However, the Born in Guangzhou Cohort Study reported that higher family SES was associated with greater risk of macrosomia, independent of potential confounders [26]. Interestingly, our findings were of decreased risk of macrosomia and LGA in pregnancies of women in professional or managerial occupations (parameters of high SES). One reason for the inconsistency may be the different parameters of SES used in previous studies: the Shaanxi cohort study used the Demographic and Health Survey household wealth index to measure SES [9]; the Born in Guangzhou Cohort Study used a principal component analysis based on ten socioeconomic indicators to calculate family SES [26], making it difficult to compare these studies.

Many researchers have used some SES indicators as proxies for others. In our study, the correlations between each indicator of SES showed no multicollinearity, suggesting that the level of maternal educational level or maternal occupation may not be proportionate to participant and partner's income. This is consistent with the suggestion from other studies that the SES indicators of education level, occupation and income may not be interchangeable $[16,27,28]$. Our results showed that the effect of maternal education on PROM was only apparent at univariate and initial adjustment level. We did not found a statistically significant association between after further adjustment for income and occupation. A similar finding pertained when we examined the associations between $\mathrm{C}$-section and parameters of SES; no significant association was found after adjustment for all cofounders. This finding indicated that education and income may less important as independent factors than other risks related to pregnancy outcomes, and is in accord with other studies [10,29-31]. Although most health studies that consider SES treat socioeconomic characteristics as potential confounders of relationships between other variables and health, others explicitly examine relationships between SES and health, to better understand the associations [27]. Despite such endeavours, the effect of SES on pregnancy outcomes has yet to be adequately explained.

This study had several limitations. Firstly, this study was conducted in the First Affiliated Hospital of Chongqing Medical University and the Chongqing Health Center for Women and Children which were located in the main urban area of Chongqing, thus limiting generalizability of our results to rural populations. Secondly, information on socio-demographic factors was obtained using face to face questionnaires, which might have introduced biases or errors. In China, income is considered private for individuals, thus participant and partner's income may have been underreported. Maternal occupation was obtained at enrollment, it may have changed during the pregnancy. Finally, SES is a complex and multidimensional concept comprising a range of factors encompassing education, occupation, income, 
family background, home ownership, car ownership and place of residence and neighborhood. We lacked such comprehensive data for our study population and so our analysis was based on the three generic indicators of SES: education, income and occupation.

\section{Conclusions}

Our results showed that only maternal occupation was observed to be independently associated with macrosomia and LGA after full adjustment for potential confounders. In contrast, education and income were not associated with adverse pregnancy outcomes. Future studies should consider validating our findings using more comprehensive measures, to understand the mechanisms underlying the link between SES and adverse pregnancy outcomes, and why maternal occupation may play a more important role than education and income.

\section{Declarations}

\section{Abbreviations}

SES: socioeconomic status, CLIMB: Complex Lipids in Mothers and Babies, GDM: gestational diabetes mellitus, $\mathrm{PIH}$ : pregnancy-induced hypertension, $\mathrm{PE}$ : pre-eclampsia, PROM: premature rupture of membrane, PTB: preterm birth, LBW: low birth weight, LGA: large for gestational age, SGA: small for gestational age ORs: odds ratios, Cls: confidence intervals

\section{Ethics approval and consent to participate}

Written informed consent was obtained from all participants at enrollment. Ethical approval for this study was provided by the Ethics Committee of Chongqing Medical University (2014034). The study was conducted in accordance with the principles in the Declaration of Helsinki 1964 and the International Conference on Harmonisation Good Clinical Practice E6 (ICH-GCP). This trial was prospectively registered with the Chinese Clinical Trial Register (ChiCTR-IOR-16007700).

\section{Consent for publication}

Not applicable.

\section{Availability of data and materials}

The datasets used and/or analysed during the current study are available from the corresponding author on reasonable request.

\section{Competing interests}


The authors declare that they have no competing interests.

\section{Funding}

This study was supported by the New Zealand Primary Growth Partnership post farm gate dairy programme, funded by Fonterra Co-operative Group Ltd, New Zealand and the New Zealand Ministry for Primary Industries. The funding bodies played no role in designing the study; in collecting, analyzing, or interpreting data; or in writing the manuscript.

\section{Authors' contributions}

YYX, WZ, TLH, CC, HZ, HBQ and PB conceived and designed research; TZ and YTC recruited the patients. TZ, QT, QT, TK and RYZ performed data checking, cleaning and the statistical analysis. ZT wrote the draft of manuscript text and prepared the tables. YYX, WZ, TLH, CC, HZ, HBQ and PB revised the manuscript. All authors read and approved the final version of the article.

\section{Acknowledgements}

We would like to thank the women who participated in this study and the health-care staff who were involved in data collection at the First Affiliated Hospital of Chongqing Medical University and the Chongqing Health Center for Women and Children.

\section{References}

1.Galobardes B, Shaw M, Lawlor DA, Lynch JW, Davey Smith G: Indicators of socioeconomic position (part 1). J Epidemiol Community Health 2006, 60(1):7-12.

2.Michael S. Kramer, Louise SeÂ guin, Goulet JLaL: Socio-economic disparities in pregnancy outcome: why do the poor fare so poorly? Paediatric and Perinatal Epidemiology 2000, 14:194-210.

3.Joseph KS, Liston RM, Dodds L, Dahlgren L, Allen AC: Socioeconomic status and perinatal outcomes in a setting with universal access to essential health care services. Canadian Medical Association Journal 2007, 177(6):583-590.

4.Silva LM, Marianne Coolmana, Eric A. P. Steegers, Vincent W. V. Jaddoe, Henrie“" tte A. Moll, Albert Hofman, Mackenbach JP, Raat aH: Low socioeconomic status is a risk factor for preeclampsia: the Generation R Study. Journal of Hypertension 2008, 26:1200-1208.

5.van den Berg G, van Eijsden M, Vrijkotte TG, Gemke RJ: Educational inequalities in perinatal outcomes: the mediating effect of smoking and environmental tobacco exposure. PLoS One 2012, 7(5):e37002. 
6.Kim MK, Lee SM, Bae SH, Kim HJ, Lim NG, Yoon SJ, Lee JY, Jo MW: Socioeconomic status can affect pregnancy outcomes and complications, even with a universal healthcare system. Int $J$ Equity Health 2018, 17(1):2.

7.Thompson JM, Clark PM, Robinson E, D B: Risk factors for small-for-gestational-age babies: The Auckland Birthweight Collaborative Study. J Paediatr Child Health 2001, 37:369-375.

8.Raisanen S, Randell K, Nielsen HS, Gissler M, Kramer MR, Klemetti R, Heinonen S: Socioeconomic status affects the prevalence, but not the perinatal outcomes, of in vitro fertilization pregnancies. Hum Reprod 2013, 28(11):3118-3125.

9.Pei L, Kang Y, Zhao Y, Cheng Y, Yan H: Changes in Socioeconomic Inequality of Low Birth Weight and Macrosomia in Shaanxi Province of Northwest China, 2010-2013: A Cross-sectional Study. Medicine (Baltimore) 2016, 95(5):e2471.

10.Campbell EE, Gilliland J, Dworatzek PDN, De Vrijer B, Penava D, Seabrook JA: Socioeconomic Status and Adverse Birth Outcomes: A Population-Based Canadian Sample. J Biosoc Sci 2017, 50(1):102-113.

11.Sow M, Racape J, Schoenborn C, De Spiegelaere M: Is the socioeconomic status of immigrant mothers in Brussels relevant to predict their risk of adverse pregnancy outcomes? BMC Pregnancy Childbirth 2018, 18(1):422.

12.Li X, Lu J, Hu S, Cheng KK, De Maeseneer J, Meng Q, Mossialos E, Xu DR, Yip W, Zhang H et al: The primary health-care system in China. The Lancet 2017, 390(10112):2584-2594.

13. Educational Statistics in 2018

[http://en.moe.gov.cn/documents/statistics/2018/national/index_1.html]

14.Huang S, Mo TT, Norris T, Sun S, Zhang T, Han TL, Rowan A, Xia YY, Zhang H, Qi HB et al: The CLIMB (Complex Lipids In Mothers and Babies) study: protocol for a multicentre, three-group, parallel randomised controlled trial to investigate the effect of supplementation of complex lipids in pregnancy, on maternal ganglioside status and subsequent cognitive outcomes in the offspring. BMJ Open 2017, 7(10):e016637.

15.Allison PD: Logistic Regression Using the SAS System: Theory and Application: SAS Publishing; 1999.

16.Nwaru BI, Klemetti R, Kun H, Hong W, Yuan S, Wu Z, Hemminki E: Maternal socio-economic indices for prenatal care research in rural China. Eur J Public Health 2011, 22(6):776-781.

17.Song L, Shen L, Li H, Liu B, Zheng X, Zhang L, Xu S, Wang Y: Socio-economic status and risk of gestational diabetes mellitus among Chinese women. Diabet Med 2017, 34(10):1421-1427.

18.Blumenshine P, Egerter S, Barclay CJ, Cubbin C, Braveman PA: Socioeconomic disparities in adverse birth outcomes: a systematic review. Am J Prev Med 2010, 39(3):263-272. 
19.Culliney KA, Parry GK, Brown J, Crowther CA: Regimens of fetal surveillance of suspected large-forgestational-age fetuses for improving health outcomes. Cochrane Database Syst Rev 2016, 4:CD011739.

20.Kc K, Shakya S, Zhang H: Gestational diabetes mellitus and macrosomia: a literature review. Ann Nutr Metab 2015, 66 Supp/ 2:14-20.

21.Wang D, Hong Y, Zhu L, Wang X, Lv Q, Zhou Q, Ruan M, Chen C: Risk factors and outcomes of macrosomia in China: a multicentric survey based on birth data. The Journal of Maternal-Fetal \& Neonatal Medicine 2016, 30(5):623-627.

22.Yao F, Miao H, Li B, Wu Y, Zhao Q: New birthweight percentiles by sex and gestational age in Southern China and its comparison with the INTERGROWTH-21st Standard. Sci Rep 2018, 8(1):7567.

23.Pitchaya Tuntiseranee, Jorn Olsen, Virasakdi Chongsuvivatwong, Limbutara S: Socioeconomic and work related determinants of pregnancy outcome in southern Thailand. J Epidemiol Community Health 1999, 53:624-629.

24.Ahmed P, Jaakkola JJ: Maternal occupation and adverse pregnancy outcomes: a Finnish populationbased study. Occup Med (Lond) 2007, 57(6):417-423.

25.Dai LL, Mao YY, Luo XM, Shen YP: Prenatal care in combination with maternal educational level has a synergetic effect on the risk of neonatal low birth weight: new findings in a retrospective cohort study in Kunshan City, China. PLoS One 2014, 9(11):e113377.

26.Tu S, Wang AL, Tan MZ, Lu JH, He JR, Shen SY, Wei DM, Lu MS, Au Yeung SL, Xia HM et al: Family socioeconomic position and abnormal birth weight: evidence from a Chinese birth cohort. World J Pediatr 2019.

27.Paula A. Braveman, Catherine Cubbin, Susan Egerter, Sekai Chideya, Kristen S. Marchi, Marilyn Metzler, Samuel Posner: Socioeconomic Status in Health Research: One Size Does Not Fit All. JAMA 2005, 294:2879-2888.

28.Shavers VL: Measurement of Socioeconomic Status in Health Disparities Research. JOURNAL OF THE NATIONAL MEDICAL ASSOCIATION2007, 99(9):1013-1023.

29.Elizabeth M. Barbeau, Nancy Krieger, Mah-Jabeen Soobader: Working Class Matters: Socioeconomic Disadvantage, Race/Ethnicity, Gender, and Smoking in NHIS 2000. American Journal of Public Health 2000, 94(2):269-278.

30.J. Morrison, J. M. Najman, G. M. Williams, J. D. Keeping, Andersen MJ: Socio-Economic Status and Pregnancy Outcome: An Australian Study. British Journal of Obstetrics and Gynaecology 1989, 96(3):298-307. 
31.Leung JY, Leung GM, Schooling CM: Socioeconomic disparities in preterm birth and birth weight in a non-Western developed setting: evidence from Hong Kong's 'Children of 1997' birth cohort. J Epidemiol Community Health 2016, 70(11):1074-1081.

\section{Tables}

Table 1. Diagnostic criteria of pregnancy outcomes

\begin{tabular}{ll}
\hline $\begin{array}{l}\text { Pregnancy } \\
\text { outcomes }\end{array}$ & Diagnostic criteria \\
\hline GDM & $\begin{array}{l}\text { A 75g oral glucose tolerance (OGTT) at 24-28 weeks, fasting, 1 and 2 h plasma glucose } \geq 5.1, \\
\geq 10.0, \geq 8.5 \mathrm{mmol} / \mathrm{L}, \text { respectively }\end{array}$ \\
PROM & Rupture of membranes before the onset of labor \\
\hline PTB & Delivery before 37 weeks \\
\hline Macrosomia & Birth weight $\geq 4000 \mathrm{~g}$ \\
\hline LBW & Birth weight $<2500 \mathrm{~g}$ \\
\hline LGA & Birth weight $>90$ th percentile of weight for a specific gestational age \\
\hline SGA & Birth weight $<10$ th percentile of weight for a specific gestational age \\
\hline
\end{tabular}

Abbreviation: GDM gestational diabetes mellitus, PIH pregnancy-induced hypertension, PE pre-eclampsia, PROM premature rupture of membrane, PTB preterm birth, LBW low birth weight, LGA large for gestational age, SGA small for gestational age

Table 2. Characteristics of the study participants and their newborns and frequency of pregnancy outcomes 


\begin{tabular}{|c|c|}
\hline \multicolumn{2}{|l|}{ Characteristic } \\
\hline \multicolumn{2}{|l|}{ Maternal characteristics } \\
\hline Maternal age (mean $\pm s d$, years) & $28.7 \pm 3.6$ \\
\hline BMI $\left(\right.$ mean $\left.\pm \mathrm{sd}, \mathrm{kg} / \mathrm{m}^{2}\right)$ & $21.5 \pm 2.9$ \\
\hline Nationality (\% Han) & 98.7 \\
\hline Marital status (\% married) & 97.9 \\
\hline Primiparity (\%) & 77.5 \\
\hline History of abortion (\%) & 47.3 \\
\hline Smoking or drinking during pregnancy (\%) & 0.4 \\
\hline \multicolumn{2}{|l|}{ Maternal educational level } \\
\hline Low educational level (\% 12 years or less) & 9.5 \\
\hline Medium educational level (\% 12-17 years) & 79.7 \\
\hline High educational level (\% more than 17 years) & 10.8 \\
\hline \multicolumn{2}{|l|}{ Participant and partner's income } \\
\hline$<7000 y u a n /$ month (\% Low income) & 21.3 \\
\hline 7000-10000yuan/month (\% Middle income) & 36.8 \\
\hline 10000-16000yuan/month (\%Middle-high income) & 30.2 \\
\hline$>16000 y u a n / m o n t h$ (\% High income) & 11.7 \\
\hline \multicolumn{2}{|l|}{ Maternal occupation } \\
\hline No occupation or student (\%) & 21.7 \\
\hline Workers/technicians/clerical/service occupations (\%) & 48.6 \\
\hline Managers/professionals (\%) & 29.7 \\
\hline \multicolumn{2}{|l|}{ Neonatal characteristics } \\
\hline Gestational age at delivery (mean \pm sd, week) & $39.4 \pm 1.5$ \\
\hline Birthweight (mean $\pm \mathrm{sd}, \mathrm{g})^{\mathrm{a}}$ & $3310.7 \pm 436.9$ \\
\hline Birthlength (mean $\pm \mathrm{sd}, \mathrm{cm})^{\mathrm{b}}$ & $49.7 \pm 1.9$ \\
\hline \multicolumn{2}{|l|}{ Newborn sex $(n, \%)^{\mathrm{C}}$} \\
\hline Male & $663(52.2)$ \\
\hline Female & $608(47.8)$ \\
\hline \multicolumn{2}{|l|}{ Pregnancy outcomes } \\
\hline GDM (n, \%) & $325(25.5)$ \\
\hline $\operatorname{PROM}(\mathrm{n}, \%)$ & $313(24.6)$ \\
\hline C-section (n, \%) & $474(37.2)$ \\
\hline PTB (n, \%) & $36(2.8)$ \\
\hline Macrosomia (n, \%) & $67(5.3)$ \\
\hline LBW $(n, \%)$ & $32(2.5)$ \\
\hline LGA (n, \%) & $116(9.1)$ \\
\hline SGA (n, \%) & $94(7.4)$ \\
\hline
\end{tabular}

Abbreviations: BMI body mass index, GDM gestational diabetes mellitus, PROM premature rupture of membrane, C-section cesarean section, PTB preterm birth, LBW low birth weight, LGA large for gestational age, SGA small for gestational age
a: Missing 11 data of birthweight
b: Missing 30 data of birthlength
c: Missing 2 data of newborn sex 
Table 3. Odds ratios (ORs) and 95\% confidence intervals (CI) for associations between maternal educational levels and adverse pregnancy outcomes

\begin{tabular}{|c|c|c|c|c|c|}
\hline $\begin{array}{l}\text { Pregnancy } \\
\text { outcomes }\end{array}$ & Education & No. (\%) & $\begin{array}{l}\text { Unadjusted OR } \\
(95 \% \mathrm{CI})\end{array}$ & $\begin{array}{l}\text { Model I OR } \\
(95 \% \mathrm{CI})\end{array}$ & $\begin{array}{l}\text { Model II OR } \\
(95 \% \mathrm{CI})\end{array}$ \\
\hline \multirow[t]{3}{*}{$\overline{\mathrm{GDM}}$} & 1 & $29(8.9)$ & 1 (reference) & 1 (reference) & 1 (reference) \\
\hline & 2 & $\begin{array}{l}262 \\
(80.6)\end{array}$ & $1.11(0.71,1.72)$ & $1.09(0.69,1.72)$ & $1.12(0.70,1.80)$ \\
\hline & 3 & $34(10.5)$ & $1.04(0.59,1.83)$ & $0.97(0.54,1.77)$ & $1.03(0.55,1.92)$ \\
\hline \multirow[t]{3}{*}{ PROM } & 1 & $19(6.1)$ & 1 (reference) & 1 (reference) & 1 (reference) \\
\hline & 2 & $\begin{array}{l}263 \\
(84.0)\end{array}$ & $1.88(1.13,3.13) *$ & $1.73(1.03,2.91) *$ & $1.67(0.98,2.84)$ \\
\hline & 3 & 31 (9.9) & $1.56(0.83,2.93)$ & $1.36(0.71,2.60)$ & $1.31(0.66,2.58)$ \\
\hline \multirow[t]{3}{*}{ C-section } & 1 & $47(9.9)$ & 1 (reference) & 1 (reference) & 1 (reference) \\
\hline & 2 & $\begin{array}{l}384 \\
(81.0)\end{array}$ & $0.96(0.65,1.41)$ & $0.98(0.65,1.48)$ & $1.11(0.73,1.71)$ \\
\hline & 3 & $43(9.1)$ & $0.71(0.43,1.19)$ & $0.72(0.42,1.24)$ & $0.85(0.48,1.51)$ \\
\hline \multirow[t]{3}{*}{ PTB } & 1 & $3(5.2)$ & 1 (reference) & 1 (reference) & 1 (reference) \\
\hline & 2 & $45(77.6)$ & $1.83(0.56,5.97)$ & $1.80(0.54,5.93)$ & $1.47(0.43,5.08)$ \\
\hline & 3 & $10(17.2)$ & $3.07(0.83,11.44)$ & $2.89(0.76,11.05)$ & $1.88(0.45,7.77)$ \\
\hline \multirow[t]{3}{*}{ Macrosomia } & 1 & $8(11.9)$ & 1 (reference) & 1 (reference) & 1 (reference) \\
\hline & 2 & $51(76.1)$ & $0.75(0.35,1.62)$ & $0.86(0.39,1.91)$ & $1.06(0.47,2.42)$ \\
\hline & 3 & $8(11.9)$ & $0.87(0.32,2.39)$ & $1.22(0.42,3.58)$ & $1.86(0.60,5.73)$ \\
\hline \multirow[t]{3}{*}{ LBW } & 1 & $3(9.4)$ & 1 (reference) & 1 (reference) & 1 (reference) \\
\hline & 2 & $24(75.0)$ & $0.95(0.28,3.21)$ & $0.78(0.15,4.19)$ & $0.84(0.14,5.10)$ \\
\hline & 3 & $5(15.6)$ & $1.48(0.35,6.32)$ & $1.22(0.17,8.98)$ & $1.60(0.17,14.82)$ \\
\hline \multirow[t]{3}{*}{ LGA } & 1 & $11(9.5)$ & 1 (reference) & 1 (reference) & 1 (reference) \\
\hline & 2 & $93(80.2)$ & $1.01(0.52,1.94)$ & $1.14(0.58,2.25)$ & $1.35(0.67,2.72)$ \\
\hline & 3 & $12(10.3)$ & $0.95(0.40,2.24)$ & $1.25(0.51,3.06)$ & $1.67(0.65,4.25)$ \\
\hline \multirow[t]{3}{*}{ SGA } & 1 & $11(11.7)$ & 1 (reference) & 1 (reference) & 1 (reference) \\
\hline & 2 & 76 (80.9) & $0.81(0.42,1.57)$ & $0.68(0.34,1.33)$ & $0.80(0.39,1.62)$ \\
\hline & 3 & $7(7.4)$ & $0.53(0.20,1.43)$ & $0.39(0.14,1.07)$ & $0.50(0.17,1.43)$ \\
\hline
\end{tabular}

Values are odds ratios (95\% confidence intervals), $* p<0.05$

Model 1 was adjusted for maternal age, BMI, Han nationality, primiparity, history of abortion, gestational age at delivery, newborn sex; GDM, PROM, C-section and preterm birth was not adjusted for gestational age at delivery, newborn sex

Model 2 was additionally adjusted for participant and partner's income, maternal occupation; GDM, PROM, Csection and preterm birth was not adjusted for gestational age at delivery, newborn sex

Abbreviations: OR Odds ratios, CI confidence intervals, GDM gestational diabetes mellitus, PROM premature rupture of membrane, C-section cesarean section, PTB preterm birth, LBW low birth weight, LGA large for gestational age, SGA small for gestational age 
Maternal educational level:

- Low educational level (12 years or less)

- Medium educational level (12-17 years)

- High educational level (more than 17 years)

Table 4. Odds ratios (ORs) and 95\% confidence intervals (CI) for associations between participant and partner's income and pregnancy outcomes

\begin{tabular}{|c|c|c|c|c|c|}
\hline $\begin{array}{l}\text { Pregnancy } \\
\text { outcomes }\end{array}$ & Income & No. (\%) & $\begin{array}{l}\text { Unadjusted OR } \\
\text { (95\%CI) }\end{array}$ & $\begin{array}{l}\text { Model I OR } \\
(95 \% \mathrm{CI})\end{array}$ & $\begin{array}{l}\text { Model II OR } \\
(95 \% \mathrm{CI})\end{array}$ \\
\hline \multirow[t]{4}{*}{$\overline{\mathrm{GDM}}$} & 1 & $67(20.6)$ & 1 (reference) & 1 (reference) & 1 (reference) \\
\hline & 2 & $\begin{array}{l}120 \\
(36.9)\end{array}$ & $1.05(0.74,1.48)$ & $1.00(0.70,1.42)$ & $1.00(0.69,1.43)$ \\
\hline & 3 & $98(30.2)$ & $1.04(0.73,1.49)$ & $1.04(0.72,1.50)$ & $1.06(0.72,1.56)$ \\
\hline & 4 & $40(12.3)$ & $1.12(0.71,1.76)$ & $1.05(0.66,1.68)$ & $1.07(0.66,1.73)$ \\
\hline \multirow[t]{4}{*}{ PROM } & 1 & $67(21.4)$ & 1 (reference) & 1 (reference) & 1 (reference) \\
\hline & 2 & $\begin{array}{l}118 \\
(37.7)\end{array}$ & $1.02(0.72,1.45)$ & $1.03(0.73,1.46)$ & $0.94(0.65,1.34)$ \\
\hline & 3 & $96(30.7)$ & $1.01(0.71,1.45)$ & $0.99(0.69,1.42)$ & $0.87(0.60,1.28)$ \\
\hline & 4 & $32(10.2)$ & $0.83(0.52,1.34)$ & $0.82(0.51,1.34)$ & $0.75(0.46,1.23)$ \\
\hline \multirow[t]{4}{*}{ C-section } & 1 & $109(23)$ & 1 (reference) & 1 (reference) & 1 (reference) \\
\hline & 2 & $\begin{array}{l}179 \\
(37.8)\end{array}$ & $0.92(0.68,1.25)$ & $0.86(0.62,1.18)$ & $0.88(0.64,1.22)$ \\
\hline & 3 & $\begin{array}{l}125 \\
(26.4)\end{array}$ & $0.72(0.52,0.99) *$ & $0.70(0.50,0.97) *$ & $0.75(0.53,1.06)$ \\
\hline & 4 & $61(12.9)$ & $1.03(0.69,1.55)$ & $0.96(0.63,1.46)$ & $1.03(0.67,1.60)$ \\
\hline \multirow[t]{4}{*}{ PTB } & 1 & $10(17.2)$ & 1 (reference) & 1 (reference) & 1 (reference) \\
\hline & 2 & $18(31.0)$ & $1.04(0.47,2.29)$ & $1.02(0.46,2.25)$ & $0.96(0.43,2.14)$ \\
\hline & 3 & $18(31.0)$ & $1.28(0.58,2.83)$ & $1.26(0.57,2.79)$ & $1.08(0.47,2.46)$ \\
\hline & 4 & $12(20.7)$ & $2.29(0.96,5.43)$ & $2.19(0.92,5.23)$ & $1.85(0.75,4.55)$ \\
\hline \multirow[t]{4}{*}{ Macrosomia } & 1 & $16(23.9)$ & 1 (reference) & 1 (reference) & 1 (reference) \\
\hline & 2 & $29(43.3)$ & $1.05(0.56,1.97)$ & $1.05(0.55,2.02)$ & $1.18(0.61,2.30)$ \\
\hline & 3 & $17(25.4)$ & $0.74(0.37,1.49)$ & $0.81(0.39,1.67)$ & $0.94(0.44,2.01)$ \\
\hline & 4 & $5(7.5)$ & $0.55(0.20,1.54)$ & $0.57(0.19,1.67)$ & $0.63(0.21,1.88)$ \\
\hline \multirow[t]{4}{*}{ LBW } & 1 & $4(12.5)$ & 1 (reference) & 1 (reference) & 1 (reference) \\
\hline & 2 & 15 (46.9) & $2.21(0.72,6.71)$ & $2.47(0.63,9.65)$ & $2.60(0.65,10.31)$ \\
\hline & 3 & 7 (21.9) & $1.24(0.36,4.28)$ & $1.06(0.21,5.30)$ & $1.11(0.21,5.83)$ \\
\hline & 4 & $6(18.8)$ & $2.80(0.78,10.09)$ & $1.40(0.25,7.88)$ & $1.21(0.20,7.48)$ \\
\hline \multirow[t]{4}{*}{ LGA } & 1 & $26(22.4)$ & 1 (reference) & 1 (reference) & 1 (reference) \\
\hline & 2 & $44(37.9)$ & $0.98(0.59,1.62)$ & $0.95(0.56,1.60)$ & $1.02(0.59,1.73)$ \\
\hline & 3 & $32(27.6)$ & $0.86(0.50,1.47)$ & $0.91(0.52,1.59)$ & $1.02(0.57,1.83)$ \\
\hline & 4 & $14(12.1)$ & $0.98(0.49,1.93)$ & $0.99(0.49,2.00)$ & $1.06(0.51,2.19)$ \\
\hline \multirow[t]{4}{*}{ SGA } & 1 & $26(27.7)$ & 1 (reference) & 1 (reference) & 1 (reference) \\
\hline & 2 & $33(35.1)$ & $0.71(0.42,1.22)$ & $0.70(0.41,1.21)$ & $0.73(0.42,1.28)$ \\
\hline & 3 & 27 (28.7) & $0.71(0.41,1.25)$ & $0.68(0.38,1.20)$ & $0.76(0.42,1.39)$ \\
\hline & 4 & $8(8.5)$ & $0.53(0.24,1.21)$ & $0.50(0.22,1.15)$ & $0.57(0.25,1.33)$ \\
\hline
\end{tabular}


Values are odds ratios (95\% confidence intervals), $* p<0.05$

Model 1 was adjusted for maternal age, BMI, Han nationality, primiparity, history of abortion, gestational age at delivery, newborn sex; GDM, PROM, C-section and preterm birth was not adjusted for gestational age at delivery, newborn sex

Model 2 was additionally adjusted for participant and partner's income, maternal occupation; GDM, PROM, Csection and preterm birth was not adjusted for gestational age at delivery, newborn sex

Abbreviations: OR Odds ratios, CI confidence intervals, GDM gestational diabetes mellitus, PROM premature rupture of membrane, C-section cesarean section, PTB preterm birth, LBW low birth weight, LGA large for gestational age, SGA small for gestational age

Participant and partner's income:

- <7000yuan/month (Low income)

- 7000-10000yuan/month (Middle income)

- 10000-16000yuan/month (Middle-high income)

- $>16000 y u a n / m o n t h$ (High income)

Table 5. Odds ratios (ORs) and 95\% confidence intervals (CI) for associations between maternal occupation and pregnancy outcomes 


\begin{tabular}{|c|c|c|c|c|c|}
\hline $\begin{array}{l}\text { Pregnancy } \\
\text { outcomes } \\
\end{array}$ & Occupation & No. (\%) & $\begin{array}{l}\text { Unadjusted OR } \\
(95 \% \mathrm{CI})\end{array}$ & $\begin{array}{l}\text { Model I OR } \\
(95 \% \mathrm{CI})\end{array}$ & $\begin{array}{l}\text { Model II OR } \\
(95 \% \mathrm{CI})\end{array}$ \\
\hline \multirow[t]{3}{*}{ GDM } & 1 & $68(20.9)$ & 1 (reference) & 1 (reference) & 1 (reference) \\
\hline & 2 & $\begin{array}{l}171 \\
(52.6)\end{array}$ & $1.17(0.84,1.62)$ & $1.06(0.75,1.48)$ & $1.03(0.73,1.46)$ \\
\hline & 3 & $86(26.5)$ & $0.90(0.63,1.30)$ & $0.86(0.59,1.26)$ & $0.84(0.56,1.25)$ \\
\hline \multirow[t]{3}{*}{ PROM } & 1 & $57(18.2)$ & 1 (reference) & 1 (reference) & 1 (reference) \\
\hline & 2 & $\begin{array}{l}157 \\
(50.2)\end{array}$ & $1.31(0.93,1.84)$ & $1.31(0.93,1.86)$ & $1.29(0.90,1.85)$ \\
\hline & 3 & 99 (31.6) & $1.36(0.94,1.98)$ & $1.32(0.90,1.92)$ & $1.30(0.87,1.94)$ \\
\hline \multirow[t]{3}{*}{ C-section } & 1 & $\begin{array}{l}113 \\
(23.8)\end{array}$ & 1 (reference) & 1 (reference) & 1 (reference) \\
\hline & 2 & $\begin{array}{l}236 \\
(49.8)\end{array}$ & $0.89(0.67,1.19)$ & $0.79(0.58,1.07)$ & $0.83(0.60,1.13)$ \\
\hline & 3 & $\begin{array}{l}125 \\
(26.4)\end{array}$ & $0.71(0.52,0.98) *$ & $0.70(0.50,0.98) *$ & $0.75(0.53,1.08)$ \\
\hline \multirow[t]{3}{*}{ PTB } & 1 & $10(17.2)$ & 1 (reference) & 1 (reference) & 1 (reference) \\
\hline & 2 & $20(34.5)$ & $0.89(0.41,1.92)$ & $0.83(0.38,1.80)$ & $0.75(0.33,1.68)$ \\
\hline & 3 & $28(48.3)$ & $2.13(1.02,4.46) *$ & $2.04(0.97,4.31)$ & $1.72(0.77,3.85)$ \\
\hline \multirow[t]{3}{*}{ Macrosomia } & 1 & $22(32.8)$ & 1 (reference) & 1 (reference) & 1 (reference) \\
\hline & 2 & $31(46.3)$ & $0.61(0.35,1.07)$ & $0.65(0.36,1.17)$ & $0.64(0.35,1.19)$ \\
\hline & 3 & $14(20.9)$ & $0.44(0.22,0.88) *$ & $0.47(0.23,0.97) *$ & $0.44(0.21,0.95) *$ \\
\hline \multirow[t]{3}{*}{ LBW } & 1 & $6(18.8)$ & 1 (reference) & 1 (reference) & 1 (reference) \\
\hline & 2 & $13(40.6)$ & $0.97(0.36,2.57)$ & $0.85(0.26,2.82)$ & $0.89(0.25,3.20)$ \\
\hline & 3 & $13(40.6)$ & $1.60(0.60,4.27)$ & $0.89(0.25,3.17)$ & $0.89(0.22,3.65)$ \\
\hline \multirow[t]{3}{*}{ LGA } & 1 & $30(25.9)$ & 1 (reference) & 1 (reference) & 1 (reference) \\
\hline & 2 & $64(55.2)$ & $0.95(0.60,1.50)$ & $0.92(0.57,1.48)$ & $0.87(0.53,1.43)$ \\
\hline & 3 & $22(19.0)$ & $0.51(0.29,0.90) *$ & $0.53(0.29,0.95) *$ & $0.48(0.26,0.89) *$ \\
\hline \multirow[t]{3}{*}{ SGA } & 1 & $24(25.5)$ & 1 (reference) & 1 (reference) & 1 (reference) \\
\hline & 2 & $47(50.0)$ & $0.86(0.52,1.44)$ & $0.84(0.50,1.42)$ & $0.95(0.55,1.64)$ \\
\hline & 3 & $23(24.5)$ & $0.68(0.38,1.23)$ & $0.62(0.34,1.14)$ & $0.75(0.39,1.43)$ \\
\hline
\end{tabular}

Values are odds ratios (95\% confidence intervals), ${ }^{*} p<0.05$

Model 1 was adjusted for maternal age, BMI, Han nationality, primiparity, history of abortion, gestational age at delivery, newborn sex; GDM, PROM, C-section and preterm birth was not adjusted for gestational age at delivery, newborn sex

Model 2 was additionally adjusted for maternal educational level, participant and partner's income; GDM, PROM, C-section and preterm birth was not adjusted for gestational age at delivery, newborn sex

Abbreviations: OR Odds ratios, CI confidence intervals, GDM gestational diabetes mellitus, PROM premature rupture of membrane, C-section cesarean section, PTB preterm birth, LBW low birth weight, LGA large for gestational age, SGA small for gestational age

Maternal occupation

- No occupation or student 
- Workers/technicians/clerical/service occupations

- Managers/professional

\section{Supplementary Files}

This is a list of supplementary files associated with this preprint. Click to download.

- Additionalfile1Xia.docx 